\title{
The Periodic Boundary Value Problem for the Weakly Dissipative $\mu$-Hunter-Saxton Equation
}

\author{
Zhengyong Ouyang, Xiangdong Wang, and Haiwu Rong \\ Department of Mathematics, Foshan University, Guangdong Province 528000, China \\ Correspondence should be addressed to Zhengyong Ouyang; zyouyang_math@163.com
}

Received 17 May 2015; Accepted 22 July 2015

Academic Editor: Stephen C. Anco

Copyright (C) 2015 Zhengyong Ouyang et al. This is an open access article distributed under the Creative Commons Attribution License, which permits unrestricted use, distribution, and reproduction in any medium, provided the original work is properly cited.

We study the periodic boundary value problem for the weakly dissipative $\mu$-Hunter-Saxton equation. We establish the local wellposedness in Besov space $B_{2,1}^{3 / 2}$, which extends the previous regularity range to the critical case.

\section{Introduction}

In this paper we consider the weakly dissipative $\mu$-HunterSaxton $(\mu \mathrm{HS})$ equation with periodic boundary condition [1]:

$$
\begin{aligned}
& \mu(u)_{t}-u_{x x t}+2 \mu(u) u_{x}+\lambda\left(\mu(u)-u_{x x}\right) \\
& =2 u_{x} u_{x x}+u u_{x x x}, \quad t \geq 0, x \in \mathbb{S}, \\
& u(0, x)=u_{0}(x), \quad x \in \mathbb{S},
\end{aligned}
$$

where $\mathbb{S}=\mathbb{R} / 2 \pi \mathbb{Z}, \mu(u)=\int_{\mathbb{S}} u \mathrm{~d} x, \lambda \geq 0$, and the term $\lambda\left(\mu(u)-u_{x x}\right)$ models energy dissipation.

When $\lambda=0$ and $\mu(u)=0$, (1) is the Hunter-Saxton equation [2]

$$
u_{x x t}+2 u_{x} u_{x x}+u u_{x x x}=0 .
$$

The Hunter-Saxton (HS) equation is an asymptotic equation for rotators in liquid crystals and modeling the propagation of weakly nonlinear orientation waves in a massive nematic liquid crystal [2]. The HS equation has a bi-Hamiltonian structure [3,4] and is completely integrable [5]. The initial value problem of the HS equation also has been studied extensively; see $[2,6,7]$. We refer to [8-12] for the weak solution to the HS equation. When $\mu(u)=0$, the global existence and blow-up phenomena for the dissipative HS equation can be found in [13].

When $\lambda=0$, (1) becomes the $\mu$-HS equation

$$
\mu(u)_{t}-u_{x x t}+2 \mu(u) u_{x}=2 u_{x} u_{x x}+u u_{x x x},
$$

which is derived and studied in [14]. Khesin et al. [14] showed that the $\mu$-HS equation describes the geodesic flow on $D^{s}(\mathbb{S})$ with the right-invariant metric given at the identity by the inner product

$$
(u, v)=\mu(u) \mu(v)+\int_{\mathbb{S}} u_{x} v_{x} \mathrm{~d} x .
$$

Moreover, if interactions of rotators and an external magnetic field are allowed, then the $\mu$-HS equation can be viewed as a natural generalization of the rotator equation. The periodic peaked solution and the multipeakons solution to $\mu$-HS equation were showed in $[14,15]$.

Another important model which possesses some similar structures to the $\mu$-HS equation is the Camassa-Holm $(\mathrm{CH})$ equation:

$$
u_{t}-u_{x x t}+3 u u_{x}=2 u_{x} u_{x x}+u u_{x x x} .
$$

The Camassa-Holm $(\mathrm{CH})$ equation was derived independently by Fokas and Fuchssteiner in [16] and by Camassa and Holm in [17]. Fokas and Fuchssteiner derived (6) in studying completely integrable generalizations of the $\mathrm{KdV}$ equation with bi-Hamiltonian structures, while Camassa and Holm proposed (6) to describe the unidirectional propagation of shallow water waves over a flat bottom. In [18], Constantin and Lannes proved that the $\mathrm{CH}$ equation, as a model for the propagation of shallow water waves, is valid approximations to the governing equations for water waves. The relation 
between the $\mathrm{CH}$ and $\mathrm{HS}$ equation is that the HS equation can be viewed as the high-frequency limit of the $\mathrm{CH}$ equation [5]. In [17], Camassa and Holm discovered the bi-Hamiltonian structure of $\mathrm{CH}$, which ensures the existence of an infinite number of conservation laws. The integrability of $\mathrm{CH}$ (as an infinite-dimensional Hamiltonian system) was studied in $[19,20]$. Moreover, the $\mathrm{CH}$ equation is such an equation that exhibits both phenomena of soliton interaction (peaked soliton solutions) and wave breaking (the solution remains bounded while its slope becomes unbounded in finite time [21]). The global existence of strong and weak solution and blow-up phenomena for the $\mathrm{CH}$ equation can be found in [21-25].

In general, it is almost impossible to avoid energy dissipation in the real world. Therefore, it is reasonable to study the model with energy dissipation. For example, the weakly dissipative $\mathrm{CH}$ equation and weakly dissipative DP equation have been studied in [26] and [27-29], respectively.

Recently, Liu and Yin [1] suggested the weakly dissipative $\mu$-HS equation. They established the local well-posedness in Sobolev spaces $H^{s}(s>3 / 2)$, obtained the global existence, and studied the blow-up scenario. Motivated by the previous work, in this paper, we aim to prove the local well-posedness for (1) (2) in the critical Besov space $B_{2,1}^{3 / 2}$.

Integrating (1) with respect to $x \in \mathbb{S}$ yields $(\mathrm{d} / \mathrm{d} t) \mu(u)=$ $-\lambda \mu(u)$, which implies that

$$
\begin{aligned}
\mu(u)=\mu\left(u_{0}\right) e^{-\lambda t} & =\mu_{0} e^{-\lambda t} \\
& \text { where } \mu_{0}:=\mu\left(u_{0}\right)=\int_{\mathbb{S}} u_{0}(x) \mathrm{d} x .
\end{aligned}
$$

Now we denote $A=\left(\mu-\partial_{x}^{2}\right)$ and reformulate (1), (2) as the nonlocal form:

$$
\begin{aligned}
\partial_{t} u+u \partial_{x} u+F(u) & =0, \quad t \geq 0, \quad x \in \mathbb{S} \\
u(0, x) & =u_{0}(x), \quad x \in \mathbb{S}
\end{aligned}
$$

where $F(u)=F_{1}(u)+F_{2}(u)+F_{3}(u)$ with

$$
\begin{aligned}
& F_{1}(u)=2 \partial_{x} A^{-1}\left(\mu_{0} e^{-\lambda t} u\right), \\
& F_{2}(u)=\frac{1}{2} \partial_{x} A^{-1}\left(u_{x}^{2}\right), \\
& F_{3}(u)=\lambda u .
\end{aligned}
$$

For the inverse operator $A^{-1}$, the relation $v=A^{-1} w$ is given explicitly by $[14,30]$,

$$
\begin{aligned}
v(x)= & \left(\frac{x^{2}}{2}-\frac{x}{2}+\frac{13}{12}\right) \mu(w)+\int_{0}^{1} \int_{0}^{y} w(s) \mathrm{d} s \mathrm{~d} y \\
& -\int_{0}^{x} \int_{0}^{y} w(s) \mathrm{d} s \mathrm{~d} y \\
& +\int_{0}^{1} \int_{0}^{y} \int_{0}^{s} w(r) \mathrm{d} r \mathrm{~d} s \mathrm{~d} y .
\end{aligned}
$$

In terms of Green's function, we see that $v=A^{-1} w$ can be also expressed as $[14,30]$

$$
v(x)=A^{-1} w=g * w=\int_{0}^{1} g(x-y) w(y) \mathrm{d} y
$$

where $g(x)$ is the periodic extension of $\widetilde{g}(x)$ with $\widetilde{g}(x)=$ $(1 / 2) x(x-1)+13 / 12, x \in[0,1]$. Besides, as $A^{-1}$ and $\partial_{x}$ commute, then

$$
\begin{aligned}
A^{-1} \partial_{x} w(x)= & \left(x-\frac{1}{2}\right) \int_{0}^{1} w(x) \mathrm{d} x-\int_{0}^{x} w(y) \mathrm{d} y \\
& +\int_{0}^{1} \int_{0}^{x} w(y) \mathrm{d} y \mathrm{~d} x, \\
A^{-1} \partial_{x}^{2} w(x)= & -w(x)+\int_{0}^{1} w(x) \mathrm{d} x .
\end{aligned}
$$

We will show that the nonlocal terms $F(u)$ map $B_{2,1}^{3 / 2}$ to $B_{2,1}^{3 / 2}$ (see Lemma 10) and therefore we can expect the local wellposedness of (1) in $B_{2,1}^{3 / 2}$.

Notations. We use $\lesssim, \gtrsim$, and $\approx$ to denote estimates that hold up to some universal constant. $C$ is also a generic constant that may assume different values in different lines. $\mathscr{D}(\mathbb{S})$ is the space of all infinitely differentiable functions on $\mathbb{S}$ and $\mathscr{D}^{\prime}(\mathbb{S})$ is its dual space. All function spaces are over $\mathbb{S}$ and we drop $\mathbb{S}$ in our notations of function spaces if there is no ambiguity.

The main results of this paper are as follows.

Theorem 1. When $u_{0} \in B_{2,1}^{3 / 2}$, we have the following results:

(1) There exists a $T=T\left(u_{0}\right)>0$ such that (1), (2) have a unique solution $u \in C\left([0, T] ; B_{2,1}^{3 / 2}\right) \cap C^{1}\left([0, T] ; B_{2,1}^{1 / 2}\right)$ which depends continuously on the initial data $u_{0}$. Furthermore, for some $C>0$, we have

$$
\|u(t)\|_{B_{2,1}^{3 / 2}} \leq 2\left\|u_{0}\right\|_{B_{2,1}^{3 / 2}}
$$

$$
\text { for } 0 \leq t \leq T=\frac{1}{4 C\left\|u_{0}\right\|_{B_{2,1}^{3 / 2}}} \text {. }
$$

(2) If $u, v$ are the solutions with initial data $u_{0}, v_{0} \in B(0$, $R) \subset B_{2,1}^{3 / 2}$, respectively, then

$$
\begin{aligned}
& \|u(t)-v(t)\|_{B_{2,1}^{q}} \leq\left\|u_{0}-v_{0}\right\|_{B_{2, \infty}^{1 / 2}}^{\alpha}, \\
& \qquad \leq \leq t \leq \widetilde{T}=\frac{1}{(4 C R+4 C \lambda)},
\end{aligned}
$$

where $\alpha=(3 / 2-q) \exp \left\{-C_{R}\right\}$ with the constant $C_{R}>0$ depending on the radius $R$ and $1 / 2 \leq q<3 / 2$. That is to say, the solution map $u_{0} \mapsto u(t, \cdot)$ defined by (1), (2) is Hölder continuous as a map from $B(0, R) \subset B_{2,1}^{3 / 2}$, with the $B_{2, \infty}^{1 / 2}$ topology, to $C\left([0, \widetilde{T}], B_{2,1}^{q}\right)$. 
Now we outline the proof of Theorem 1 . We construct a sequence of the approximate solutions $\left\{u_{n}\right\}$. We need to prove that $\left\{u_{n}\right\}$ is a Cauchy sequence. However, in the critical case, the following estimate

$$
\|F(u)-F(v)\|_{B_{2,1}^{2 / 1}} \leqslant\|u-v\|_{B_{2,1}^{2 / 1}}
$$

cannot be obtained due to the lack of appropriate product estimate in $B_{2,1}^{-1 / 2}$ (this is one of the significant differences between the critical case $s=3 / 2$ and the supercritical case $s>3 / 2$ ). Therefore we will use a product estimate in $B_{2, \infty}^{-1 / 2}$ obtained in Danchin's cerebrated paper [31] (see Lemma 7) and the Log interpolation inequality (see Lemma 8 ) to find

$$
\begin{aligned}
& \|F(u)-F(v)\|_{B_{2, \infty}^{1 / 2}} \lesssim\|u-v\|_{B_{2,1}^{1 / 2}} \\
& \quad \leq C\|u-v\|_{B_{2, \infty}^{1 / 2}} \ln \left(e+\frac{\|u-v\|_{R_{2, \infty}^{3 / 2}}}{\|u-v\|_{B_{2, \infty}^{1 / 2}}}\right) .
\end{aligned}
$$

Then we prove the convergence of $\left\{u_{n}\right\}$ in $B_{2, \infty}^{1 / 2}$ firstly and then to obtain the convergence in $B_{2,1}^{1 / 2}$ by interpolation. To this end, we will use the Osgood lemma (Lemma 9) to estimate $\|u-v\|_{B_{2 \infty}^{1 / 2}}$. Our idea is motivated by $[32,33]$, where the authors used the Osgood lemma to studied the bfamily equation and the modified Camassa-Holm equation, respectively.

The remainder of this paper is organized as follows. In the next section, we state some preliminaries. We prove Theorem 1 in Section 3.

\section{Preliminaries}

Now we state some preliminaries on the Littlewood-Paley theory. We refer to [34-36] for the elementary properties of them.

Let $\chi, \phi$ be two smooth radial functions satisfying $0 \leq \chi$, $\varphi \leq 1, \operatorname{supp} \chi \subset\{\xi \in \mathbb{R}:|\xi| \leq 4 / 3\}, \operatorname{supp} \varphi \subset\{\xi \in \mathbb{R}: 3 / 4 \leq$ $|\xi| \leq 8 / 3\}$, and

$$
\begin{aligned}
\chi(\xi)+\sum_{j \in \mathbb{N}} \varphi\left(2^{-j} \xi\right) & =1, & & \forall \xi \in \mathbb{R}, \\
\sum_{j \in \mathbb{Z}} \varphi\left(2^{-j} \xi\right) & =1, & & \forall \xi \in \mathbb{R} \backslash\{0\}, \\
\operatorname{supp} \varphi\left(2^{-j} \cdot\right) \cap \operatorname{supp} \varphi\left(2^{-j^{\prime}} \cdot\right) & =\varnothing & & \text { if }\left|j-j^{\prime}\right| \geq 2, \\
\operatorname{supp} \varphi(\cdot) \cap \operatorname{supp} \varphi\left(2^{-j} \cdot\right) & =\varnothing & & \text { if } j \geq 1 .
\end{aligned}
$$

We decompose $u \in \mathscr{D}^{\prime}(\mathbb{S})$ into Fourier series; that is, $u=$ $(1 / 2 \pi) \sum_{\xi \in \mathbb{Z}} \mathscr{F}_{x} u(\xi) e^{i x \xi}$, where $\mathscr{F}_{x} u(\xi)=\int_{\mathbb{S}} e^{-i x \xi} u(x) \mathrm{d} x$ is the Fourier transform and the inverse transform is given by $\mathscr{F}_{x}^{-1} u(x)=(1 / 2 \pi) \sum_{\xi \in \mathbb{Z}} u(\xi) e^{i x \xi}$. Now we define the periodic dyadic blocks as

$$
\begin{aligned}
\Delta_{-1} u & =\mathscr{F}_{x}^{-1} \chi \mathscr{F}_{x} u=\frac{1}{2 \pi} \sum_{\xi \in \mathbb{Z}} \chi(\xi)\left(\mathscr{F}_{x} u\right)(\xi) e^{i x \xi}, \\
\Delta_{j} u & =\mathscr{F}_{x}^{-1} \varphi\left(2^{-j} \xi\right) \mathscr{F}_{x} u \\
& =\frac{1}{2 \pi} \sum_{\xi \in \mathbb{Z}} \varphi\left(2^{-j} \xi\right)\left(\mathscr{F}_{x} u\right)(\xi) e^{i x \xi}, \quad \text { if } j \geq 0 .
\end{aligned}
$$

Then we define the low frequency cut-off $S_{j}$ as $S_{j} u=$ $\sum_{i=-1}^{j-1} \Delta_{i} u$. Direct computation implies that, for any $1 \leq p \leq$ $\infty$, we have the quasi-orthogonality properties:

$$
\begin{aligned}
\Delta_{i} \Delta_{j} u \equiv 0, & \text { if }|i-j| \geq 2, \\
\Delta_{j}\left(S_{i-1} u \Delta_{i} v\right) \equiv 0, & \text { if }|i-j| \geq 5 .
\end{aligned}
$$

Furthermore, for all $u \in L^{p},\left\|\Delta_{i} u\right\|_{L^{p}} \leq\|u\|_{L^{p}}$ and $\left\|S_{j} u\right\|_{L^{p}} \leq$ $C\|u\|_{L^{p}}$.

Definition 2 (Besov spaces). Let $s \in \mathbb{R}, 1 \leq p$, and $r \leq+\infty$. The nonhomogeneous Besov space $B_{p, r}^{s}(\mathbb{S})$ is defined by

$$
B_{p, r}^{s}(\mathbb{S})=\left\{f \in \mathscr{D}^{\prime}(\mathbb{S}):\|f\|_{B_{p, r}^{s}(\mathbb{S})}<\infty\right\},
$$

where $\|f\|_{B_{p, r}^{s}(\$)}=\left\|2^{j s} \Delta_{j} f\right\|_{l^{r}\left(L^{p}\right)}=\left\|\left(2^{j s}\left\|\Delta_{j} f\right\|_{L^{p}}\right)_{j \geq-1}\right\|_{l^{r}}$.

The following lemma summarizes some useful properties of $B_{p, r}^{s}$.

Lemma 3 (see [34-36]). Let $s \in \mathbb{R}$ and $1 \leq p, r, p_{j}, r_{j} \leq \infty$, $j=1,2$. Then the following properties hold:

(1) Consider $B_{p_{1}, r_{1}}^{s_{1}} \hookrightarrow B_{p_{2}, r_{2}}^{s_{2}}$ if $p_{1} \leq p_{2}$; consider $r_{1} \leq r_{2}$ and $s_{2}=s_{1}-\left(1 / p_{1}-1 / p_{2}\right)$.

$B_{p, r_{1}}^{s_{1}} \hookrightarrow B_{\mathrm{p}, r_{2}}^{s_{2}}$ is locally compact if $s_{2}<s_{1} ; r_{1} \leq r_{2}$.

(2) $\forall s>0, B_{p, r}^{s} \cap L^{\infty}$ is a Banach algebra. $B_{p, r}^{s}$ is a Banach algebra $\Leftrightarrow B_{p, r}^{s} \hookrightarrow L^{\infty} \Leftrightarrow s>1 / p$ (or $s \geq 1 / p$ and $r=1)$.

(3) Consider $\forall \theta \in[0,1], s=\theta s_{1}+(1-\theta) s_{2}$, and

$$
\|f\|_{B_{p, r}^{s}} \leq C\|f\|_{B_{p, r}^{s_{1}}}^{\theta}\|f\|_{B_{p, r}^{s_{2}}}^{1-\theta}, \quad \forall f \in B_{p, r}^{s_{1}} \cap B_{p, r}^{s_{2}} .
$$

(4) Consider $\forall \theta \in(0,1), s_{1}>s_{2}, s=\theta s_{1}+(1-\theta) s_{2}$, and

$$
\|u\|_{B_{p, 1}^{s}} \leq \frac{C(\theta)}{s_{1}-s_{2}}\|u\|_{B_{p, \infty}^{s_{1}}}^{\theta}\|u\|_{B_{p, \infty}^{2}}^{1-\theta}, \quad \forall u \in B_{p, \infty}^{s_{1}} .
$$

(5) If $\left\{u_{n}\right\}_{n \in \mathbb{N}}$ is bounded in $B_{p, r}^{s}$ and $u_{n}$ converges to $u$ in $\mathscr{D}^{\prime}(\mathbb{S})$, then $u \in B_{p, r}^{s}$ and

$$
\|u\|_{B_{p, r}^{s}} \leq \liminf _{n \rightarrow \infty}\left\|u_{n}\right\|_{B_{p, r}^{s}} .
$$

For $A=\left(\mu-\partial_{x}^{2}\right)$, we see that $\mathscr{F}_{x}\left(A^{-1} f\right)=(1 /(\delta(\xi)+$ $\left.\left.\xi^{2}\right)\right) \mathscr{F}_{x} f$, where $\delta(\xi)=1$ if $\xi=0$ and $\delta(\xi)=0$ if $\xi \neq 0$. Therefore, we see that if $s \in \mathbb{R}, f \in B_{p, r}^{s}$ with $1 \leq p, r \leq \infty$, 
then $\left\|A^{-1} f\right\|_{B_{p, r}^{s+2}} \leqslant\|f\|_{B_{p, r}^{s}}$, which means that $A^{-1}$ is bounded from $B_{p, r}^{s}$ into $B_{p, r}^{s+2}$.

Now we recall some results of the transport equation (see [34] for the details).

Lemma 4 (a priori estimates). Let $1 \leq p, r \leq \infty$, and $s>$ $-\min \{1 / p, 1-1 / p\}$. Assume that $f_{0} \in B_{p, r}^{s}, F \in L^{1}\left(0, T ; B_{p, r}^{s}\right)$, and $\partial_{x} v \in L^{1}\left(0, T ; B_{p, r}^{s-1}\right)$ if $s>1+1 / p$ or $\partial_{x} v \in L^{1}\left(0, T ; B_{p, r}^{1 / p} \cap\right.$ $\left.L^{\infty}\right)$ otherwise. If $f \in L^{\infty}\left(0, T ; B_{p, r}^{s}\right) \cap C\left([0, T] ; \mathscr{D}^{\prime}(\mathbb{S})\right)$ solves the $1 D$ linear transport equation

$$
\begin{gathered}
f_{t}+v f_{x}=F, \quad t>0, \quad x \in \mathbb{S}, \\
f(0, x)=f_{0}, \quad x \in \mathbb{S},
\end{gathered}
$$

then there exists a constant $C$ depending only on $s, p, r$ such that the following statements hold:
(1) For all $t \in[0, T]$,

$$
\begin{aligned}
\|f\|_{B_{p, r}^{s}} \leq & \left\|f_{0}\right\|_{B_{p, r}^{s}}+\int_{0}^{t}\|F(\tau)\|_{B_{p, r}^{s}} \mathrm{~d} \tau \\
& +C \int_{0}^{t} V^{\prime}(\tau)\|f(\tau)\|_{B_{p, r}^{s}} \mathrm{~d} \tau,
\end{aligned}
$$

and hence

$$
\|f\|_{B_{p, r}^{s}} \leq e^{C V(t)}\left(\left\|f_{0}\right\|_{B_{p, r}^{s}}+\int_{0}^{t} e^{-C V(\tau)}\|F(\tau)\|_{B_{p, r}^{s}} \mathrm{~d} \tau\right),
$$

$$
V(t)= \begin{cases}\int_{0}^{t}\left\|v_{x}(\tau)\right\|_{B_{p, r}^{1 / p} \cap L^{\infty}} \mathrm{d} \tau, & \text { if } s<1+\frac{1}{p}, \\ \int_{0}^{t}\left\|v_{x}(\tau)\right\|_{B_{p, r}^{s-1}} \mathrm{~d} \tau, & \text { if } s>1+\frac{1}{p} \text { or }\left(s=1+\frac{1}{p}, r=1\right) .\end{cases}
$$

(2) If $f=v$, then (1) holds true for all $s>0$ with $V(t)=$ $\int_{0}^{t}\left\|v_{x}(\tau)\right\|_{L^{\infty}} \mathrm{d} \tau$.

Lemma 5 (existence and uniqueness). Let $p, r, s, f_{0}$, and $F$ be as in the statement of Lemma 4. Assume that $v \in$ $L^{\rho}\left(0, T ; B_{\infty, \infty}^{-M}\right)$ for some $\rho>1, M>0$, and $v_{x} \in L^{1}\left(0, T ; B_{p, r}^{s-1}\right)$ if $s>1+1 / p$ or $s=1+1 / p, r=1$, and $v_{x} \epsilon$ $L^{1}\left(0, T ; B_{p, \infty}^{1 / p} \cap L^{\infty}\right)$ if $s<1+1 / p$. Then (24) have a unique solution $f \in L^{\infty}\left(0, T ; B_{p, r}^{s}\right) \cap C\left([0, T] ; B_{p, 1}^{s^{\prime}}\right)$ for any $s^{\prime}<s$ and the inequalities of Lemma 4 hold true. Moreover, if $r<\infty$, then $f \in C\left([0, T] ; B_{p, r}^{s}\right)$.

We need the following lemmas.

Lemma 6 (Moser-type estimates; see $[34,37])$. Let $1 \leq p$, $r \leq+\infty$, and $s>0$; then

$$
\begin{array}{r}
\|f g\|_{B_{p, r}^{s}} \leq C\left(\|f\|_{B_{p, r}^{s}}\|g\|_{L^{\infty}}+\|f\|_{L^{\infty}}\|g\|_{B_{p, r}^{s}}\right) \\
\forall f, g \in B_{p, r}^{s} \cap L^{\infty} .
\end{array}
$$

Lemma 7 (see [34]). For any $f \in B_{2,1}^{-1 / 2}, g \in B_{2,1}^{1 / 2}$, there holds the product estimate

$$
\|f g\|_{B_{2, \infty}^{-1 / 2}} \lesssim\|f\|_{B_{2,1}^{-1 / 2}}\|g\|_{B_{2,1}^{1 / 2}}
$$

Lemma 8 (see [34]). There is a constant $C>0$ such that, for $s \in \mathbb{R}, \varepsilon>0$, and $1 \leq p \leq \infty$,

$$
\begin{aligned}
&\|f\|_{B_{p, 1}^{s}} \leq C \frac{1+\varepsilon}{\varepsilon}\|f\|_{B_{p, \infty}^{s}} \ln \left(e+\frac{\|f\|_{B_{p, \infty}^{s+\varepsilon}}}{\|f\|_{B_{p, \infty}^{s}}}\right) \\
& \forall f \in B_{p, \infty}^{s+\varepsilon} .
\end{aligned}
$$

Lemma 9 (Osgood lemma; see $[34,38]$ ). Let $\rho \geq 0$ be a measurable function, $\gamma>0$ a locally integrable function, and $\mu$ a continuous and increasing function. Assume that, for some nonnegative real number $c$, the function $\rho$ satisfies

$$
\rho(t) \leq c+\int_{t_{0}}^{t} \gamma\left(t^{\prime}\right) \mu\left(\rho\left(t^{\prime}\right)\right) \mathrm{d} t^{\prime} .
$$

If $c>0$, then $-\mathscr{M}(\rho(t))+\mathscr{M}(c) \leq \int_{t_{0}}^{t} \gamma\left(t^{\prime}\right) \mathrm{d} t^{\prime}$ with $\mathscr{M}=$ $\int_{x}^{1}(\mathrm{~d} r / \mu(r))$.

If $c=0$ and $\mu$ verifies the condition $\int_{0}^{1}(\mathrm{~d} r / \mu(r))=+\infty$, then the function $\rho=0$.

Now we establish some estimates on $F(u)$ and the difference of two solutions.

Lemma 10. Let F be given in (9); then there holds the estimate:

$$
\begin{aligned}
\|F(u)\|_{B_{2,1}^{3 / 2}} & \leqslant\|u\|_{B_{2,1}^{3 / 2}}+\left\|u_{x}\right\|_{L^{\infty}}\|u\|_{B_{2,1}^{3 / 2}}+\lambda\|u\|_{B_{2,1}^{3 / 2}} \\
& \lesssim\left(\|u\|_{B_{2,1}^{3 / 2}}+\lambda\right)^{2}, \quad \forall u \in B_{p, r}^{s} .
\end{aligned}
$$


Proof. Since $B_{2,1}^{1 / 2} \hookrightarrow L^{\infty}$, (32) follows from Lemma 6 easily and we omit the proof here.

Lemma 11. For any $u, v$ in $B_{2,1}^{3 / 2}$, the following estimates hold:

$$
\begin{aligned}
& \|F(u)-F(v)\|_{B_{2, \infty}^{1 / 2}} \\
& \quad \leq\left(\|u\|_{B_{2,1}^{3 / 2}}+\|v\|_{B_{2,1}^{3 / 2}}+\lambda\right)\|u-v\|_{B_{2,1}^{1 / 2}} .
\end{aligned}
$$

Proof. Equation (33) follows from (9) and Lemma 7. We omit the details here.

Lemma 12. If $u, v \in C\left([0, T] ; \mathscr{D}^{\prime}(\mathbb{S})\right) \cap L^{\infty}\left(0, T ; B_{2,1}^{3 / 2}\right)$ are two solutions to (1) for some $T>0$ with initial data $u_{0}, v_{0} \in B_{2,1}^{3 / 2}$, respectively, then, for any $s^{\prime} \in(1 / 2,3 / 2)$ and $t \in[0, T]$,

$$
\begin{aligned}
& \|u-v\|_{B_{2,1}^{s^{\prime}}} \leqslant\left\|u_{0}-v_{0}\right\|_{B_{2, \infty}^{1 / 2}}^{\theta \exp \{-C T\}}, \\
& \qquad \theta=\frac{3}{2}-s^{\prime} \in(0,1),
\end{aligned}
$$

where $C>0$ is a constant depending on $\|u\|_{L^{\infty}\left(0, T ; B_{2,1}^{3 / 2}\right)}$ and $\|v\|_{L^{\infty}\left(0, T ; B_{2,1}^{3 / 2}\right)}$.

Proof. Since $u, v$ are two solutions to (1) with initial data $u_{0}, v_{0} \in B_{2,1}^{3 / 2}$, respectively, we know that $w=u-v$ satisfies

$$
\begin{array}{r}
w_{t}+v \partial_{x} w=-w \partial_{x} u-(F(u)-F(v)), \\
w(0, x)=u_{0}-v_{0},
\end{array}
$$

where $F(\cdot)$ is given in (9). Let $U(t)=\int_{0}^{t}\left\|v_{x}(\tau)\right\|_{B_{2, \infty}^{1 / 2} \cap L^{\infty}} \mathrm{d} \tau$, by Lemma 4; we have

$$
\begin{aligned}
\|w(t)\|_{B_{2, \infty}^{1 / 2}} \leq & \left\|w_{0}\right\|_{B_{2, \infty}^{1 / 2}} e^{C U(t)} \\
& +\int_{0}^{t} e^{C U(t)-C U(\tau)}\|\widehat{F}\|_{B_{2, \infty}^{1 / 2}} \mathrm{~d} \tau,
\end{aligned}
$$

where $\widehat{F}=-(u-v) \partial_{x} u-[F(u)-F(v)]$. Since $u, v \in L^{\infty}(0, T$; $\left.B_{2,1}^{3 / 2}\right)$, we see that

$$
\|v(t)\|_{B_{2,1}^{3 / 2}}+\|u(t)\|_{B_{2,1}^{3 / 2}} \leq M
$$

$$
0 \leq t \leq T \text {, for some } M>0 \text {, }
$$

and therefore we have $U(t) \lesssim 1$. Since $B_{2,1}^{s} \hookrightarrow B_{2, \infty}^{\mathcal{S}}$, by Lemma 11 and (37), we arrive at

$$
\begin{aligned}
& \|F(u)-F(v)\|_{B_{2, \infty}^{1 / 2}} \\
& \quad \leq\left(\|u\|_{B_{2,1}^{1 / 2}}+\|v\|_{B_{2,1}^{1 / 2}}+\lambda\right)\|u-v\|_{B_{2,1}^{1 / 2}} \lesssim\|u-v\|_{B_{2,1}^{1 / 2}} . \\
& \left\|b(u-v) \partial_{x} u\right\|_{B_{2, \infty}^{1 / 2}} \lesssim\|u-v\|_{B_{2,1}^{1 / 2}}\|u\|_{B_{2,1}^{3 / 2}} \lesssim\|u-v\|_{B_{2,1}^{1 / 2}} .
\end{aligned}
$$

Hence $\|\widehat{F}\|_{B_{2, \infty}^{1 / 2}} \leqslant\|u-v\|_{B_{2,1}^{1 / 2}}$. From Lemma $8(\varepsilon=1, s=1 / 2)$ and (37), we obtain that

$$
\begin{aligned}
\|\widehat{F}\|_{B_{2, \infty}^{1 / 2}} \leqslant\|u-v\|_{B_{2,1}^{1 / 2}} & \\
\leqslant\|u-v\|_{B_{2, \infty}^{1 / 2}} \ln \left(e+\frac{M}{\|u-v\|_{B_{2, \infty}^{1 / 2}}}\right), & \\
& 0 \leq t \leq T .
\end{aligned}
$$

Consequently, for all $t \in[0, T]$,

$$
\begin{aligned}
& \|w(t)\|_{B_{2, \infty}^{1 / 2}} \\
& \leqslant\|w(0)\|_{B_{2, \infty}^{1 / 2}} \\
& \quad+C \int_{0}^{t}\|w(\tau)\|_{B_{2, \infty}^{1 / 2}} \ln \left(e+\frac{M}{\|w(\tau)\|_{B_{2, \infty}^{1 / 2}}}\right) \mathrm{d} \tau .
\end{aligned}
$$

Using the fact that $\ln (e+M / x) \leq \ln (e+1)(1-\ln (x /$ $M))$ for $x \in(0, M]$, we can infer from (40) that

$$
\begin{aligned}
& \frac{\|w(t)\|_{B_{2, \infty}^{1 / 2}}}{M} \leqslant \frac{\|w(0)\|_{B_{2, \infty}^{1 / 2}}}{M} \\
&+C \int_{0}^{t} \frac{\|w\|_{B_{2, \infty}^{1 / 2}}}{M}\left(1-\ln \frac{\|w\|_{B_{2, \infty}^{1 / 2}}}{M}\right) \mathrm{d} \tau, \\
& \forall t \in[0, T] .
\end{aligned}
$$

Thanks to Lemma 9, we obtain

$$
\frac{\|w(t)\|_{B_{2, \infty}^{1 / 2}}}{e M} \lesssim\left(\frac{\|w(0)\|_{B_{2, \infty}^{1 / 2}}}{e M}\right)^{\exp \{-C T\}} .
$$

Therefore, we have

$$
\|w(t)\|_{B_{2, \infty}^{1 / 2}} \lesssim\|w(0)\|_{B_{2, \infty}^{1 / 2}}^{\exp \{-C T\}} .
$$

If $1 / 2<s^{\prime}<3 / 2$, by interpolation, the embedding $B_{2,1}^{1 / 2} \hookrightarrow$ $B_{2, \infty}^{1 / 2}$, and (37), we obtain

$$
\begin{aligned}
\|w\|_{B_{2,1}^{s^{\prime}}} & \leq\|w\|_{B_{2, \infty}^{1 / 2}}^{\theta}\|w\|_{B_{2, \infty}^{3 / 2}}^{1-\theta} \\
& \leq\|w\|_{B_{2, \infty}^{1 / 2}}^{\theta}\left(\|u\|_{B_{2,1}^{3 / 2}}+\|v\|_{B_{2,1}^{3 / 2}}\right)^{1-\theta} \lesssim\|w\|_{B_{2, \infty}^{1 / 2}}^{\theta},
\end{aligned}
$$

where $\theta=3 / 2-s^{\prime}$. Using (43), we obtain that

$$
\|w\|_{B_{2,1}^{s^{\prime}}} \leqslant\|w\|_{B_{2, \infty}^{1 / 2}}^{\theta} \lesssim\|w(0)\|_{B_{2, \infty}^{1 / 2}}^{\theta \exp \{-C T\}} .
$$

We finish the proof by combining (43) and (45).

Lemma 13 (see [31]). Denote $\overline{\mathbb{N}}=\mathbb{N} \cup\{\infty\}$. Let $\left\{a_{k}\right\}_{k \in \overline{\mathbb{N}}}$ be a sequence of continuous bounded functions on $[0, T] \times \mathbb{S}$ with 
$\sup _{k \in \overline{\mathbb{N}}}\left\|a_{k}(t)\right\|_{B_{2,1}^{3 / 2}} \leq \alpha(t)$ for some $\alpha(t) \in L^{1}(0, T)$ and $T>0$. Assume that $v_{k}$ solves

$$
\begin{aligned}
\partial_{t} v_{k}+a_{k} \partial_{x} v_{k} & =g, \quad t \geq 0, \quad x \in \mathbb{S}, \\
v_{k}(0) & =v_{0}, \quad x \in \mathbb{S},
\end{aligned}
$$

with $v_{0} \in B_{2,1}^{3 / 2}, g \in L^{1}\left(0, T ; B_{2,1}^{1 / 2}\right)$. If $a_{n} \rightarrow a_{\infty}$ in $L^{1}(0, T$; $\left.B_{2,1}^{1 / 2}\right)$, then $v_{n} \rightarrow v_{\infty}$ in $C\left([0, T] ; B_{2,1}^{1 / 2}\right)$.

\section{Proof of Theorem 1}

In this section, we prove Theorem 1 by the following several steps.

3.1. Approximate Solutions and Their Uniform Bounds. Starting from $u_{1}=0$ and by induction, we define a sequence of smooth functions $\left\{u_{n}\right\}, n \in \mathbb{N}$ by solving the following transport equation iteratively:

$$
\begin{aligned}
\partial_{t} u_{n+1}+u_{n} \partial_{x} u_{n+1} & =-F\left(u_{n}\right), \\
u_{n+1}(0, x) & =S_{n+1} u_{0},
\end{aligned}
$$

where $F(u)=\sum_{1 \leq i \leq 3} F_{i}(u)$ and $F_{i}(u)$ given in (9). Since all the data belong to $B_{2,1}^{\infty}$, from Lemma 5 and by induction, we can show that, for all $n \geq 1$, the above equation has a global solution $u_{n+1}$ belonging to $C\left(\mathbb{R}^{+}, B_{2,1}^{\infty}\right)$. For $n \in \mathbb{N}$, set $U_{n}(t)=\int_{0}^{t}\left\|u_{n}\right\|_{B_{2,1}^{3 / 2}} \mathrm{~d} \tau$. Since $\left\|u_{n+1}(0, x)\right\|_{B_{2,1}^{3 / 2}} \lesssim\left\|u_{0}\right\|_{B_{2,1}^{3 / 2}}$, from the estimate (26) of Lemmas 4 and 10, we have

$$
\begin{aligned}
& \left\|u_{n+1}(t)\right\|_{B_{2,1}^{3 / 2}} \leq e^{C U_{n}(t)}\left(\left\|u_{0}\right\|_{B_{2,1}^{3 / 2}}\right. \\
& \left.+C \int_{0}^{t} e^{-C U_{n}(\tau)}\left(\left\|u_{n}\right\|_{B_{2,1}^{3 / 2}}+\lambda\right)^{2} \mathrm{~d} \tau\right) .
\end{aligned}
$$

Reset the constant $C$ on the above estimate such that

$$
\begin{gathered}
\left\|u_{n+1}(t)\right\|_{B_{2,1}^{3 / 2}}+\lambda \leq e^{C U_{n}(t)}\left(\left\|u_{0}\right\|_{B_{2,1}^{3 / 2}}+\lambda\right. \\
\left.+C \int_{0}^{t} e^{-C U_{n}(\tau)}\left(\left\|u_{n}\right\|_{B_{2,1}^{3 / 2}}+\lambda\right)^{2} \mathrm{~d} \tau\right) .
\end{gathered}
$$

Let $T>0$ such that $2 C\left(\left\|u_{0}\right\|_{B_{2,1}^{3 / 2}}+\lambda\right) T<1$; we claim that

$$
\left\|u_{n}(t)\right\|_{B_{2,1}^{3 / 2}}+\lambda \leq \frac{\left\|u_{0}\right\|_{B_{2,1}^{3 / 2}}+\lambda}{1-2 C\left(\left\|u_{0}\right\|_{B_{2,1}^{3 / 2}}+\lambda\right) t}
$$

Assume (50) is true for $n$. We now prove that it also holds true for $n+1$. Actually, by (50), we have

$$
\begin{aligned}
& e^{C U_{n}(t)-C U_{n}(\tau)} \\
& \quad \leq \exp \left\{C \int_{\tau}^{t} \frac{\left(\left\|u_{0}\right\|_{B_{2,1}^{3 / 2}}+\lambda\right)}{\left(1-2 C\left(\left\|u_{0}\right\|_{B_{2,1}^{3 / 2}}+\lambda\right) t^{\prime}\right)} \mathrm{d} t^{\prime}\right\} \\
& \quad=\left(\frac{1-2 C\left(\left\|u_{0}\right\|_{B_{2,1}^{3 / 2}}+\lambda\right) \tau}{1-2 C\left(\left\|u_{0}\right\|_{B_{2,1}^{3 / 2}}+\lambda\right) t}\right)^{1 / 2} .
\end{aligned}
$$

From the above equation, we see clearly that when $\tau=0$, $U_{n}(\tau)=0$, we have

$$
e^{C U_{n}(t)} \leq \frac{1}{\left(1-2 C\left(\left\|u_{0}\right\|_{B_{2,1}^{3 / 2}}+\lambda\right) t\right)^{1 / 2}}
$$

Combining (49), (50), (51), and (52) gives rise to

$$
\begin{aligned}
& \left\|u_{n+1}(t)\right\|_{B_{2,1}^{3 / 2}}+\lambda \\
& \leq \frac{1}{\left(1-2 C\left(\left\|u_{0}\right\|_{B_{2,1}^{3 / 2}}+\lambda\right) t\right)^{1 / 2}}\left[\left\|u_{0}\right\|_{B_{2,1}^{3 / 2}}+\lambda\right. \\
& \left.-\frac{\left(\left\|u_{0}\right\|_{B_{2,1}^{3 / 2}}+\lambda\right)}{2} \int_{0}^{t} \frac{\mathrm{d}\left(1-2 C\left(\left\|u_{0}\right\|_{B_{2,1}^{3 / 2}}+\lambda\right) \tau\right)}{\left(1-2 C\left(\left\|u_{0}\right\|_{B_{2,1}^{3 / 2}}+\lambda\right) \tau\right)^{3 / 2}}\right] \\
& \leq \frac{\left\|u_{0}\right\|_{B_{2,1}^{3 / 2}}+\lambda}{1-2 C\left(\left\|u_{0}\right\|_{B_{2,1}^{3 / 2}}+\lambda\right) t} .
\end{aligned}
$$

Hence (50) is true for $n+1$. Setting $T=1 / 4 C\left(\left\|u_{0}\right\|_{B_{2,1}^{3 / 2}}+\lambda\right)$, for $n \in \mathbb{N}$, we can conclude that $u_{n}$ exists for $0 \leq t \leq T$ and satisfies the bound

$$
\left\|u_{n}(t)\right\|_{B_{2,1}^{3 / 2}} \leq 2\left\|u_{0}\right\|_{B_{2,1}^{3 / 2}}+\lambda \leq 1, \quad 0 \leq t \leq T
$$

Furthermore, for $t \in[0, T]$, using (54) yields

$$
\left\|\partial_{t} u_{n}\right\|_{B_{2,1}^{1 / 2}} \leq\left\|u_{n}\right\|_{B_{2,1}^{1 / 2}}\left\|\partial_{x} u_{n+1}\right\|_{B_{2,1}^{1 / 2}}+\left\|F\left(u_{n}\right)\right\|_{B_{2,1}^{3 / 2}} \lesssim 1
$$

Hence we conclude that $\left\{u_{n}\right\} \subset C\left([0, T] ; B_{2,1}^{3 / 2}\right) \cap C^{1}([0, T]$; $B_{2,1}^{1 / 2}$ ) is uniformly bounded.

3.2. Convergence of the Approximate Solutions. We now prove that $\left\{u_{n}\right\}$ is a Cauchy sequence in $C\left([0, T] ; B_{2,1}^{1 / 2}\right)$. Firstly, we see that the estimate of $u_{n+m+1}-u_{n+1}$ is essential in 
the derivation of Lemma 12. Similar to Lemma 12, we obtain that, for $m, n \in \mathbb{N}$,

$$
\begin{aligned}
& \left\|\left(u_{n+m+1}-u_{n+1}\right)(t)\right\|_{B_{2, \infty}^{1 / 2}} \leq 2^{-n} \\
& +\int_{0}^{t}\left\|\left(u_{n+m}-u_{n}\right)(\tau)\right\|_{B_{2, \infty}^{1 / 2}} \\
& \cdot \ln \left(e+\frac{\left\|\left(u_{n+m}-u_{n}\right)(\tau)\right\|_{B_{2, \infty}^{3 / 2}}}{\left\|\left(u_{n+m}-u_{n}\right)(\tau)\right\|_{B_{2, \infty}^{1 / 2}}}\right) \mathrm{d} \tau,
\end{aligned}
$$

where we used the following estimate and we have (see, e.g., in [39], page 2142)

$$
\begin{aligned}
& \left\|\left(u_{n+m+1}-u_{n+1}\right)(0)\right\|_{B_{2, \infty}^{1 / 2}} \leq\left\|\left(u_{n+m+1}-u_{n+1}\right)(0)\right\|_{B_{2,1}^{1 / 2}} \\
& \quad \leq C 2^{-n}\left\|u_{0}\right\|_{B_{2,1}^{3 / 2}} .
\end{aligned}
$$

Let $H_{n, m}(t)=\left\|\left(u_{n+m}-u_{n}\right)(t)\right\|_{B_{2, \infty}^{1 / 2}}$. Since $\left\{u_{n}\right\} \subset C([0, T]$; $\left.B_{2,1}^{3 / 2}\right) \cap C^{1}\left([0, T] ; B_{2,1}^{1 / 2}\right)$ is uniformly bounded, from (56), we obtain that

$$
\begin{aligned}
& H_{n+1, m}(t) \\
& \qquad\left(2^{-n}+\int_{0}^{t} H_{n, m}(\tau) \ln \left(e+\frac{1}{H_{n, m}(\tau)}\right) \mathrm{d} \tau\right), \\
& t \in[0, T] .
\end{aligned}
$$

As the function $x \ln (e+1 / x)$ is nondecreasing, we see that $H_{n}(t) \triangleq \sup _{m \in \mathbb{N}} H_{n, m}(t)$ satisfies

$$
\begin{array}{r}
H_{n+1}(t) \lesssim\left(2^{-n}+\int_{0}^{t} H_{n}(\tau) \ln \left(e+\frac{1}{H_{n}(\tau)}\right) \mathrm{d} \tau\right) \\
t \in[0, T] .
\end{array}
$$

Let $\widetilde{H}(t)=\lim \sup _{n \rightarrow \infty} H_{n}(t)$. For any given $\varepsilon>0$, there exists an $N \in \mathbb{N}$ such that when $n>N$, we have $H_{n}(t) \leq$ $\widetilde{H}(t)+\varepsilon$, from which it follows that

$$
\begin{array}{r}
H_{n+1}(t) \\
\lesssim\left(2^{-n}+\int_{0}^{t}(\widetilde{H}(\tau)+\varepsilon) \ln \left(e+\frac{1}{\widetilde{H}(\tau)+\varepsilon}\right) \mathrm{d} \tau\right), \\
t \in[0, T], n>N .
\end{array}
$$

Hence we have

$$
\begin{aligned}
& \limsup _{n \rightarrow \infty} H_{n}(t) \leq \limsup _{n \rightarrow \infty}\left(2^{-n}\right. \\
& \left.\quad+\int_{0}^{t}(\widetilde{H}(\tau)+\varepsilon) \ln \left(e+\frac{1}{\widetilde{H}(\tau)+\varepsilon}\right) \mathrm{d} \tau\right) \\
& \quad=\int_{0}^{t}(\widetilde{H}(\tau)+\varepsilon) \ln \left(e+\frac{1}{\widetilde{H}(\tau)+\varepsilon}\right) \mathrm{d} \tau .
\end{aligned}
$$

Since $\varepsilon>0$ is arbitrary, we have

$$
\widetilde{H}(t) \lesssim \int_{0}^{t} \widetilde{H}(\tau) \ln \left(e+\frac{1}{\widetilde{H}(\tau)}\right) \mathrm{d} \tau .
$$

Since $x \ln (e+1 / x)$ is nondecreasing and $\int_{0}^{1}(1 /(x \ln (e+$ $1 / x))) \mathrm{d} x=+\infty$, we can infer from Lemma 9 that $\widetilde{H}(t)=0$; in other words, $\left\{u_{n}\right\}$ is a Cauchy sequence in $C\left([0, T] ; B_{2, \infty}^{1 / 2}\right)$. By (5) in Lemma 3, we see

$$
\begin{aligned}
\left\|u_{n+m}-u_{n}\right\|_{B_{2,1}^{1}} & \leqslant\left\|u_{n+m}-u_{n}\right\|_{B_{2, \infty}^{1 / 2}}^{1 / 2}\left\|u_{n+m}-u_{n}\right\|_{B_{2, \infty}^{3 / 2}}^{1 / 2} \\
& \lesssim\left\|u_{n+m}-u_{n}\right\|_{B_{2, \infty}^{1 / 2}}^{1 / 2} .
\end{aligned}
$$

Hence $\left\{u_{n}\right\}_{n \in \mathbb{N}}$ is actually Cauchy sequence in $C\left([0, T] ; B_{2,1}^{1}\right)$ and therefore $\left\{u_{n}\right\}$ converges to some function $u \in C([0, T]$; $\left.B_{2,1}^{1}\right)$.

3.3. Existence, Regularity, and Uniqueness of the Solution. Since $\left\|u_{n}\right\|_{B_{2,1}^{3 / 2}}$ is uniformly bounded by $2\left\|u_{0}\right\|_{B_{2,1}^{3 / 2}}$, property $(5)$ in Lemma 3 guarantees that $\|u\|_{B_{2,1}^{3 / 2}} \leq 2\left\|u_{0}\right\|_{B_{2,1}^{3 / 2}}$, which means that $u \in L^{\infty}\left(0, T ; B_{2,1}^{3 / 2}\right)$. If $s^{\prime}<1$, we have

$$
\left\|u_{n}-u\right\|_{B_{2,1}^{s^{\prime}}} \leq\left\|u_{n}-u\right\|_{B_{2,1}^{1}}
$$

If $1<s^{\prime}<3 / 2$, by interpolation again, we have

$$
\begin{aligned}
\left\|u_{n}-u\right\|_{B_{2,1}^{s^{\prime}}} & \leq\left\|u_{n}-u\right\|_{B_{2,1}^{1}}^{\theta}\left\|u_{n}-u\right\|_{B_{2,1}^{3 / 2}}^{1-\theta} \\
& \leq\left\|u_{n}-u\right\|_{B_{2,1}^{1}}^{\theta}\left\|u_{0}\right\|_{B_{2,1}^{3 / 2}}^{1-\theta}
\end{aligned}
$$

where $\theta=3-2 s^{\prime}$. From (64) and (65), we see that $u_{k} \rightarrow u$ in $C\left([0, T] ; B_{2,1}^{s^{\prime}}\right)$ for all $s^{\prime}<3 / 2$. Taking limits to (47), we can deduce that $u$ indeed solves (8). Since $r=1<\infty$, from Lemmas 5 and 10 , we know that $u \in C\left([0, T] ; B_{2,1}^{3 / 2}\right)$. By (8), we obtain that $u_{t} \in C\left([0, T] ; B_{2,1}^{1 / 2}\right)$. The uniqueness is a corollary of Lemma 12.

3.4. Hölder Continuity of the Solution Map. The continuity of the solution map can be obtained by following the steps in [31] and using Lemma 13 and we omit the details in this paper. Now we consider the Hölder continuity of the solution map. For the initial data $u_{0} \in B(0, R) \subset B_{2,1}^{3 / 2}$, by (13), we see that the lifespan $T_{u}$ of the corresponding solution $u$ to (8) satisfies

$$
T_{u}>\frac{1}{4 C\left(\left\|u_{0}\right\|_{B_{2,1}^{3 / 2}}+\lambda\right)}>\frac{1}{4 C R+4 C \lambda} \triangleq \widetilde{T}
$$

where $\widetilde{T}$ does not depend on $u$. Therefore, we can find $\widetilde{T}>0$ such that, for all $u_{0} \in B(0, R) \subset B_{2,1}^{3 / 2}$, the corresponding solution $u \in C\left([0, \widetilde{T}] ; B_{2,1}^{3 / 2}\right)$. Directly from (13) and Lemma 12, (14) is proved.

We complete the proof of Theorem 1 . 


\section{Conflict of Interests}

The authors declare that there is no conflict of interests regarding the publication of this paper.

\section{Acknowledgments}

This work is supported by the National Natural Science Foundations of China (no. 11401096) and Guangdong Province (no. 2013KJCX0189 and no. 2014KZDXM063). The authors thank the editors for their hard working and also gratefully acknowledge helpful comments and suggestions by reviewers.

\section{References}

[1] J. Liu and Z. Yin, "On the Cauchy problem of a weakly dissipative $\mu$-Hunter-Saxton equation," Annales de l'Institut Henri Poincare (C) Non Linear Analysis, vol. 31, no. 2, pp. 267279, 2014.

[2] J. K. Hunter and R. Saxton, "Dynamics of director fields," SIAM Journal on Applied Mathematics, vol. 51, no. 6, pp. 1498-1521, 1991.

[3] R. S. Johnson, "Camassa-Holm, Korteweg-de Vries and related models for water waves," Journal of Fluid Mechanics, vol. 455, pp. 63-82, 2002.

[4] P. J. Olver and P. Rosenau, "Tri-Hamiltonian duality between solitons and solitary-wave solutions having compact support," Physical Review E, vol. 53, no. 2, pp. 1900-1906, 1996.

[5] J. K. Hunter and Y. X. Zheng, "On a completely integrable nonlinear hyperbolic variational equation," Physica D. Nonlinear Phenomena, vol. 79, no. 2-4, pp. 361-386, 1994.

[6] J. Lenells, "The Hunter-Saxton equation describes the geodesic flow on a sphere," Journal of Geometry and Physics, vol. 57, no. 10, pp. 2049-2064, 2007.

[7] Z. Yin, "On the structure of solutions to the periodic HunterSaxton equation," SIAM Journal on Mathematical Analysis, vol. 36, no. 1, pp. 272-283, 2004.

[8] A. Bressan and A. Constantin, "Global solutions of the HunterSaxton equation," SIAM Journal on Mathematical Analysis, vol. 37, no. 3, pp. 996-1026, 2005.

[9] J. K. Hunter and Y. X. Zheng, "On a nonlinear hyperbolic variational equation. I. Global existence of weak solutions," Archive for Rational Mechanics and Analysis, vol. 129, no. 4, pp. 305-353, 1995.

[10] J. K. Hunter and Y. X. Zheng, "On a nonlinear hyperbolic variational equation. II. The zero-viscosity and dispersion limits," Archive for Rational Mechanics and Analysis, vol. 129, no. 4, pp. 355-383, 1995.

[11] J. Li and K. Zhang, "Global existence of dissipative solutions to the Hunter-Saxton equation via vanishing viscosity," Journal of Differential Equations, vol. 250, no. 3, pp. 1427-1447, 2011.

[12] P. Zhang and Y. Zheng, "Existence and uniqueness of solutions of an asymptotic equation arising from a variational wave equation with general data," Archive for Rational Mechanics and Analysis, vol. 155, no. 1, pp. 49-83, 2000.

[13] X. Wei and Z. Yin, "Global existence and blow-up phenomena for the periodic Hunter-Saxton equation with weak dissipation," Journal of Nonlinear Mathematical Physics, vol. 18, no. 1, pp. 139149, 2011.
[14] B. Khesin, J. Lenells, and G. Misiolek, "Generalized HunterSaxton equation and the geometry of the group of circle diffeomorphisms," Mathematische Annalen, vol. 342, no. 3, pp. 617-656, 2008

[15] J. Lenells, G. Misiolek, and F. Tiğlay, "Integrable evolution equations on spaces of tensor densities and their peakon solutions," Communications in Mathematical Physics, vol. 299, no. 1, pp. 129-161, 2010.

[16] A. S. Fokas and B. Fuchssteiner, "Symplectic structures, their Bäklund transformations and hereditray symmetries," Physica D. Nonlinear Phenomena, vol. 4, no. 1, pp. 47-66, 1981.

[17] R. Camassa and D. D. Holm, "An integrable shallow water equation with peaked solitons," Physical Review Letters, vol. 71, no. 11, pp. 1661-1664, 1993.

[18] A. Constantin and D. Lannes, "The hydrodynamical relevance of the Camassa-Holm and Degasperis-Procesi equations," Archive for Rational Mechanics and Analysis, vol. 192, no. 1, pp. 165-186, 2009.

[19] A. Constantin, "On the inverse spectral problem for the Camassa-Holm equation," Journal of Functional Analysis, vol. 155, no. 2, pp. 352-363, 1998.

[20] A. Constantin, "Quasi-periodicity with respect to time of spatially periodic finite-gap solutions of the Camassa-Holm equation," Bulletin des Sciences Mathématiques, vol. 122, no. 7, pp. 487-494, 1998.

[21] A. Constantin and J. Escher, "Wave breaking for nonlinear nonlocal shallow water equations," Acta Mathematica, vol. 181, no. 2, pp. 229-243, 1998.

[22] A. Constantin, "Existence of permanent and breaking waves for a shallow water equation: a geometric approach," Annales de l'Institut Fourier, vol. 50, no. 2, pp. 321-362, 2000.

[23] A. Constantin and J. Escher, "Well-posedness, global existence, and blow-up phenomena for a periodic quasi-linear hyperbolic equation," Communications on Pure and Applied Mathematics, vol. 51, no. 5, pp. 475-504, 1998.

[24] H. P. McKean, "Breakdown of a shallow water equation," The Asian Journal of Mathematics, vol. 2, no. 4, pp. 867-874, 1998.

[25] Z. Xin and P. Zhang, "On the weak solution to a shallow water equation," Communications on Pure and Applied Mathematics, vol. 53, no. 11, pp. 1411-1433, 2000.

[26] S. Wu and Z. Yin, "Global existence and blow-up phenomena for the weakly dissipative Camassa-Holm equation," Journal of Differential Equations, vol. 246, no. 11, pp. 4309-4321, 2009.

[27] S. Wu, J. Escher, and Z. Yin, "Global existence and blowup phenomena for a weakly dissipative Degasperis-Procesi equation," Discrete and Continuous Dynamical Systems Series B, vol. 12, no. 3, pp. 633-645, 2009.

[28] S. Wu and Z. Yin, "Blow-up and decay of the solution of the weakly dissipative Degasperis-Procesi equation," SIAM Journal on Mathematical Analysis, vol. 40, no. 2, pp. 475-490, 2008.

[29] S. Wu and Z. Yin, "Blow-up phenomena and decay for the periodic Degasperis-Procesi equation with weak dissipation," Journal of Nonlinear Mathematical Physics, vol. 15, pp. 28-49, 2008.

[30] J. Escher, M. Kohlmann, and B. Kolev, "Geometric aspects of the periodic $\mu$-Degasperis-Procesi equation," Progress in Nonlinear Differential Equations and Their Applications, vol. 80, pp. 193209, 2011.

[31] R. Danchin, "A note on well-posedness for Camassa-Holm equation," Journal of Differential Equations, vol. 192, no. 2, pp. 429-444, 2003. 
[32] H. Tang and Z. Liu, "Well-posedness of the modified CamassaHolm equation in Besov spaces," Zeitschrift für Angewandte Mathematik und Physik, 2014.

[33] H. Tang, S. Shi, and Z. Liu, "The dependences on initial data for the b-family equation in critical Besov space," Monatshefte für Mathematik, vol. 177, no. 3, pp. 471-492, 2015.

[34] H. Bahouri, J. Chemin, and R. Danchin, Fourier Analysis and Nonlinear Partial Differential Equations, Springer, Berlin, Germany, 2011.

[35] H. J. Schmeisser and H. Triebel, Topics in Fourier Analysis and Function Spaces, Wiley, Chichester, UK, 1987.

[36] H. Triebel, Theory of Function Spaces, Birkhäuser, Basel, Switzerland, 1983.

[37] J. Chemin, "Localization in Fourier space and Navier-Stokes," in Phase Space Analysis of Partial Differential Equations, CRM Series, pp. 53-136, Scuola Normale Superiore di Pisa, Pisa, Italy, 2004.

[38] J. Chemin, Perfect Incompressible Fluids, vol. 14 of Oxford Lectures Series in Mathematics and Its Applications, The Clarendon Press, Oxford University Press, New York, NY, USA, 1998.

[39] K. Yan and Z. Yin, "On the Cauchy problem for a twocomponent Degasperis-Procesi system," Journal of Differential Equations, vol. 252, no. 3, pp. 2131-2159, 2012. 


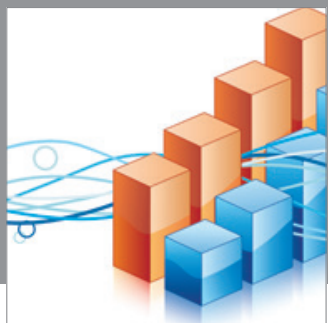

Advances in

Operations Research

mansans

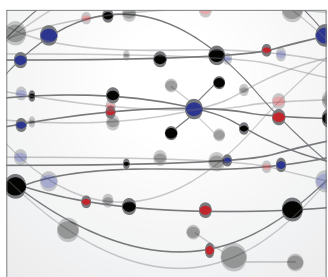

The Scientific World Journal
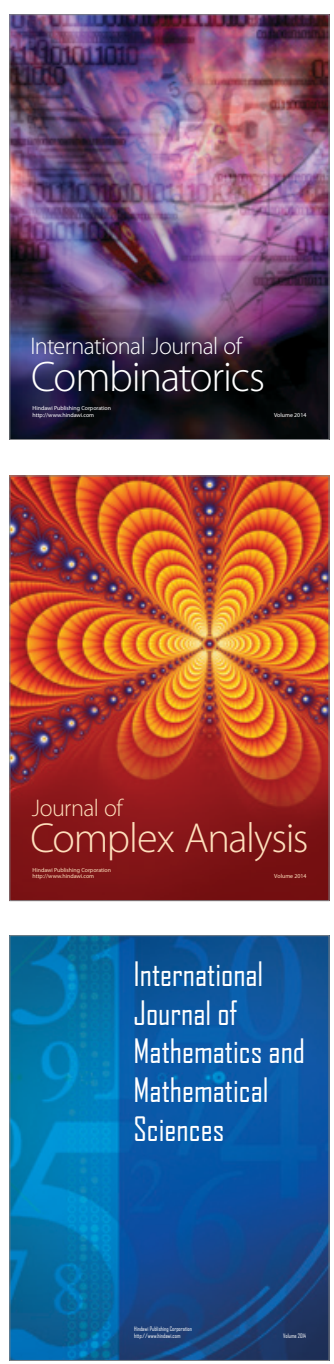
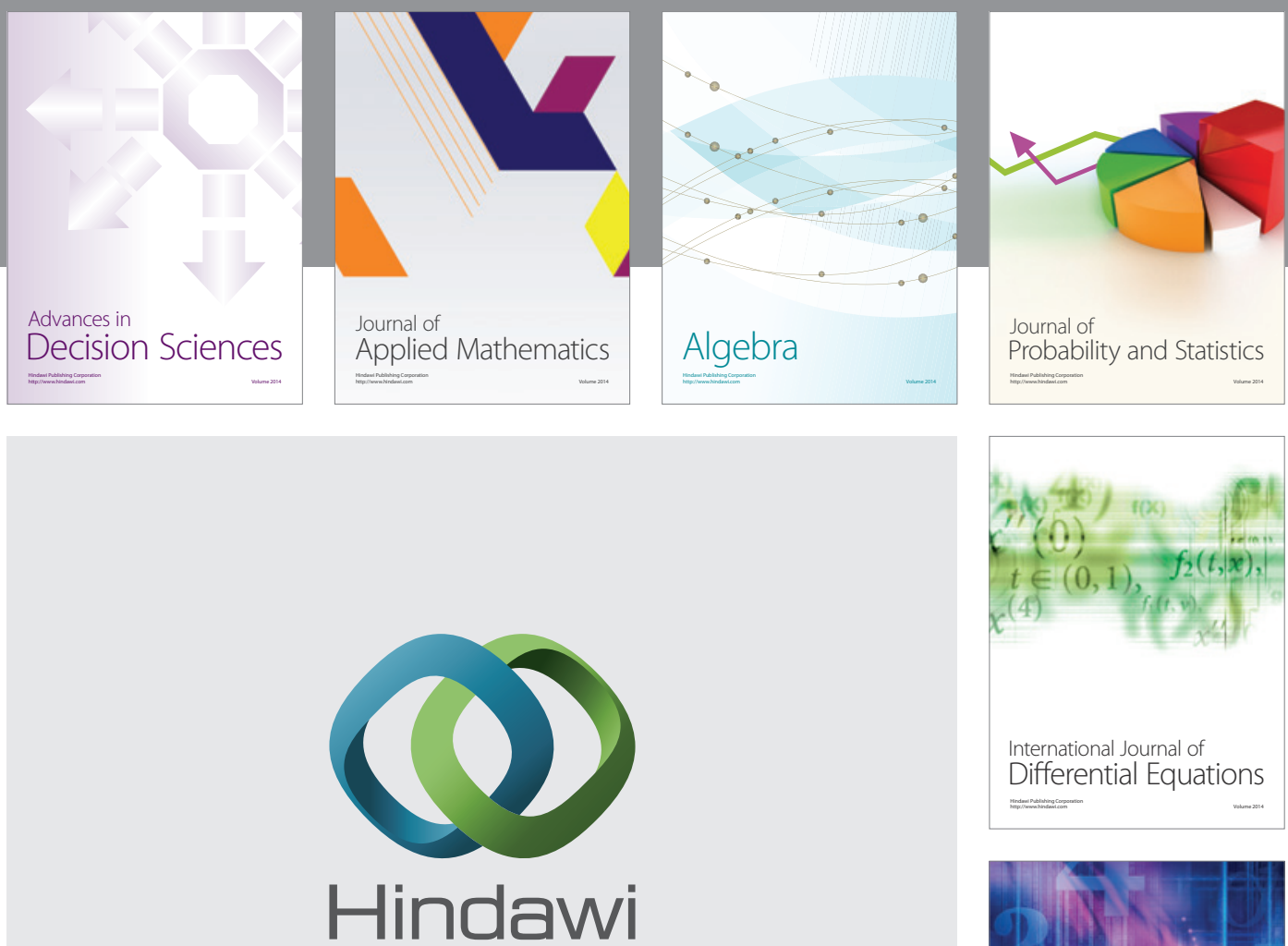

Submit your manuscripts at http://www.hindawi.com
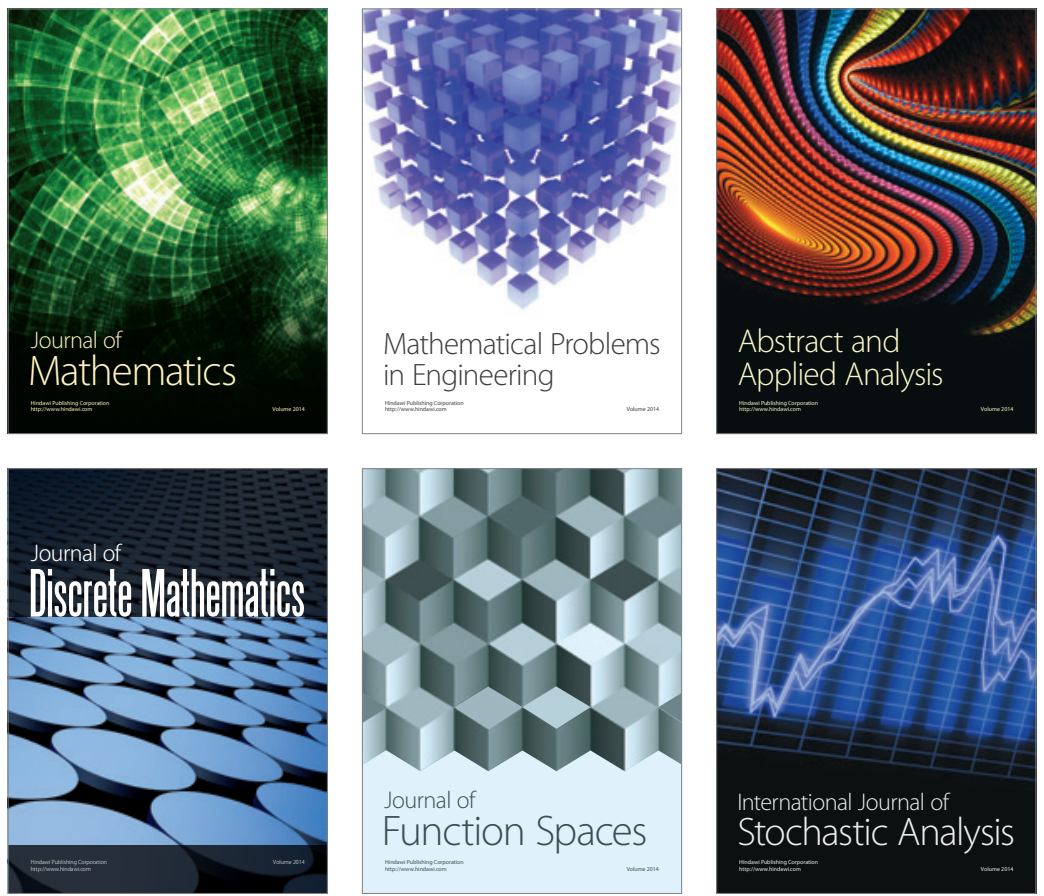

Journal of

Function Spaces

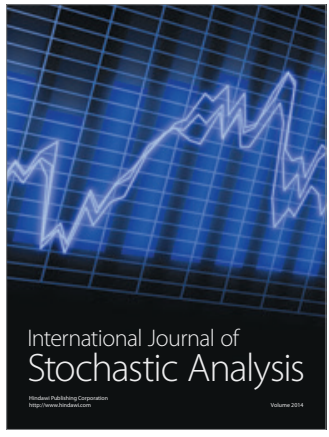

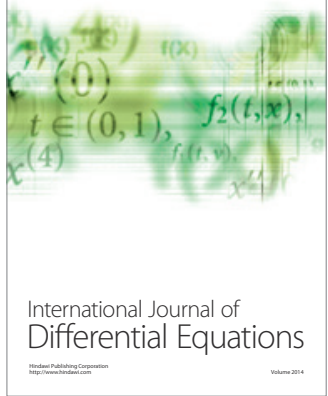
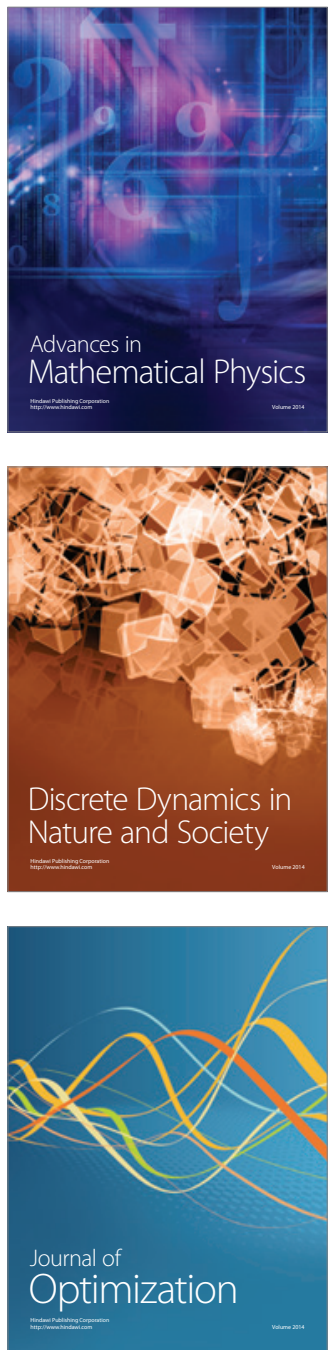